\title{
Distal realignment and patellar autologous chondrocyte implantation: mid-term results in a selected population
}

\author{
Antonio Gigante - Davide Enea · Francesco Greco • \\ Corrado Bait · Matteo Denti · Herbert Schonhuber • \\ Piero Volpi
}

Received: 14 March 2008/Accepted: 5 September 2008/Published online: 22 October 2008

(C) The Author(s) 2008. This article is published with open access at Springerlink.com

\begin{abstract}
The aim of this prospective observational study was to assess the 3-year clinical outcome of distal realignment and membrane-seeded autologous chondrocyte implantation $\left(\mathrm{MACI}^{\circledR}\right)$ in selected patients with patellofemoral malalignment and large, isolated, patellar cartilage lesions. Twelve patients (14 knees; 6 females, 6 males; mean age 31 years) with Fulkerson type II patellofemoral malalignment (lateralized and tilted patella) and Outerbridge grade III-IV isolated patellar cartilage lesions were treated. All had tibial tuberosity and trochlear sulcus $>20 \mathrm{~mm}$ on a preoperative CT scan and a cartilage defect $>3 \mathrm{~cm}^{2}$. Patients with Outerbridge grade III-IV trochlear cartilage lesions, those with rheumatic, infective or neoplastic conditions, or ligament instability, diabetes or obesity and those aged $>40$ years were excluded. Followup was at 36 months. Patients were enrolled after diagnostic arthroscopy. Cartilage was harvested and sent for culture. After a mean period of 30 days (range 25-40) patients underwent transfer of the tibial tuberosity according to Fulkerson associated with a MACI procedure. Clinical assessment was performed with the Kujala, Lysholm, Tegner and Modified Cincinnati scores. The Patient Satisfaction Survey was administered at 36 months. Consistently improved knee function and activity levels were reflected by significantly increased Kujala, Lysholm, Tegner and Modified Cincinnati scores at 36 months. The significant clinical improvement support the value of
\end{abstract}

\footnotetext{
A. Gigante $\cdot$ D. Enea $(\bowtie) \cdot$ F. Greco

Department of Orthopaedics, Polytechnic University of Marche,

Ancona, Italy

e-mail: davidenea@tiscali.it

C. Bait · M. Denti · H. Schonhuber · P. Volpi

Department of Sports Traumatology and Arthroscopic Surgery,

Galeazzi Orthopaedics Institute, Milan, Italy
}

associating distal realignment and autologous chondrocyte implantation in treating large, isolated, patellar cartilage lesions associated with patellofemoral malalignment.

Keywords MACI - Cartilage repair ·

Patellofemoral joint · Patellofemoral malalignment

\section{Introduction}

Patellar cartilage lesions are the most frequent articular cartilage defects seen in knee arthroscopic procedures [10]. The majority are Outerbridge grade III-IV lesions and can degenerate to patellofemoral osteoarthritis $[11,50]$.

Patellofemoral cartilage lesions are due to different causes including sport trauma, traffic accidents, osteochondritis dissecans, patellofemoral malalignment and idiopathic chondromalacia. Patellofemoral malalignment encompasses a number of conditions, isolated or variously associated, such as increased Q-angle, high-riding patella (patella alta), trochlear dysplasia, increased femoral anteversion, excessive tension of lateral retinaculum, absence of medial patellofemoral ligament and vastus medialis obliquus hypotrophy $[1,2,12,14,16,42]$. Such disorders lead to altered articular congruence between patella and femoral trochlea that often progresses to severe cartilage damage of one or both patellar facets. Some cartilage defects are thought to be related to patellofemoral malalignment. In such cases the malalignment may be the source of knee pain and disability, and the cause of progression to patellofemoral osteoarthritis [25, 43].

Multiple operative procedures have been devised to correct patellofemoral malalignment. Patellofemoral distal realignment, performed with various techniques, is among the most widely applied in severe cases. Anteriorization 
and medialization of the tibial tuberosity, introduced respectively by Trillat et al. [49] and Maquet [33], were eventually fused by Fulkerson into a single procedure [17]. A number of series report the results of distal realignment of the tibial tuberosity according to Fulkerson [5, 17, 36, 38, 401.

Treatment approaches to the cartilage lesions that are often seen in patellofemoral malalignment have received less attention, probably due to the poor range of cartilage repair options available until not long ago. In particular, few studies have addressed the effectiveness of combined distal realignment associated with autologous chondrocyte implantation (ACI) in repairing isolated patellar cartilage lesions.

According to Pidoriano et al. [45], anteromedialization of the tibial tubercle improves knee function and relieves anterior knee pain in 90 and $85 \%$ of type I and II patellar cartilage lesions (inferior pole and lateral facet), respectively, with lower success rates for type III (medial facet, $56 \%$ ) and type IV defects (proximal pole or diffuse lesions, $20 \%$ ).

However, A topographic study of 105 patients with anterior knee pain or simple malalignment documented that the most frequent site of cartilage lesion (71\%) is a roughly elliptical area with the major diameter parallel to the transverse axis of the patella, not affecting the upper and lower thirds of the patella, i.e., a diffuse lesion (type IV). The lateral and medial facets are affected in 7 and $21 \%$ of cases, respectively [26].

Once begun, cartilage degeneration cannot be reversed but will tend to progression [8, 35], following its own course and becoming independent of the original cause. Unless corrected, this process can undermine and even reverse the clinical outcome of the surgical realignment and eventually impair patellofemoral joint function [45].

Different operative procedures are currently applied for articular cartilage repair, including debridement, microfractures, subchondral drilling, periosteal transplantation, mosaicplasty and ACI [6, 19, 22, 28, 29, 34]. First introduced in 1994 by Brittberg et al. [7], who used a periosteal flap, the ACI technique has evolved to include the utilization of chondrocytes grown on synthetic scaffolds, thus obviating the need for harvesting periosteum and affording a less invasive surgical wound. Some recent studies have failed to document significant differences between the clinical outcomes of first-generation ACI versus its variant using a collagen membrane as a scaffold (MACI ${ }^{\circledR}$ ) $[3,39]$.

Despite the good long-term clinical outcomes [43], both techniques involve two surgical steps, the high cost of cell culture and, for MACI, the cost of the membrane itself. Their actual cost-effectiveness compared with more traditional techniques is therefore still unclear [29]. Bentley et al. have shown that ACI is far superior to mosaicplasty in repairing patellar cartilage lesions [6], while Knutsen et al. have found no significant differences in cartilage lesion repair between ACI and microfractures at 5 years [29]. However, none of the existing cartilage repair techniques have proved to be superior to the others in the longterm, although there are no works comparing different techniques for the patella. While some studies [15, 20, 23, 37,43 ] have considered the clinical outcomes of combined distal realignment and ACI, none have focused on isolated patellar lesions and precisely characterized the type and extent of the malalignment being treated.

The purpose of this study was to investigate the clinical outcomes of distal realignment combined with MACI in a subset of patients with patellofemoral malalignment and large, isolated, patellar cartilage lesions. These patients had type II patellofemoral malalignment with lateralized and tilted patella [18], tibial tuberosity and trochlear sulcus (TT-TS) $>20 \mathrm{~mm}$ [12] on preoperative CT scans and Outerbridge grade III-IV isolated patellar cartilage lesions.

\section{Materials and methods}

\section{Inclusion criteria}

In this prospective observational study we included 14 knees (two bilateral procedures) operated on between December 2000 and May 2004 at two different centers in Italy. All patients had type II patellofemoral malalignment according to Fulkerson (lateralized and tilted patella) [18] associated with large, isolated Outerbridge grade III or IV patellar cartilage lesions.

A lateralized patella is one with a positive congruence angle persisting beyond $10^{\circ}$ of knee flexion, whereas a tilted patella is one with a patellar tilt angle $<8^{\circ}$ on CT between $0^{\circ}$ and $30^{\circ}$ of flexion $[18,21,46]$. A large lesion was one $>3 \mathrm{~cm}^{2}$; an isolated patellar cartilage lesion was one not associated with an Outerbridge grade III or IV trochlear cartilage lesion.

Only patients with TT-TS $>20 \mathrm{~mm}[4,12]$ on preoperative CT scans were included (Fig. 1).

Malalignment and lesion size and grade were confirmed by diagnostic arthroscopy prior to cartilage harvesting. Patients $>40$ years old and those suffering from ligament instability, diabetes, obesity, rheumatologic, infective or neoplastic conditions were excluded. Follow-up was at 36 months.

Surgical technique

After diagnostic arthroscopy, performed to assess precisely the extent of patellofemoral tracking, confirmed the lesion's size and the feasibility of MACI and 


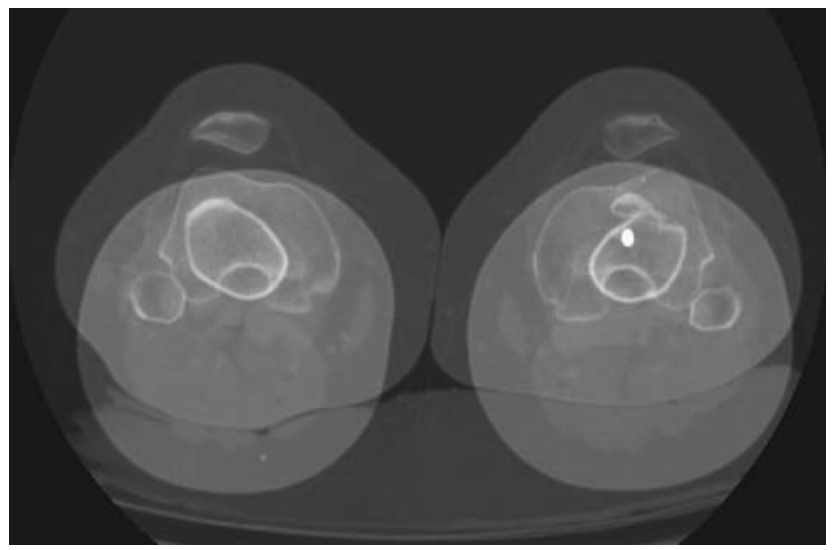

Fig. 1 Superimposed CT scans of the patellofemoral joints of the same patient taken at the midpatellar level and at the proximal end of the tibial tuberosity. Tomograms were measured for the distance (in millimeter) between anterior tibial tuberosity and trochlear sulcus (TT-TS). Images show anteromedialization of the left tuberosity compared with the untreated contralateral knee

patellofemoral distal realignment, $300-500 \mathrm{mg}$ of cartilage was harvested from the non-articulating portion of the femoral notch and then sent to the laboratory (Genzyme, Denmark) for culture.

After about 30 days (range 25-40), patients underwent MACI and patellofemoral distal realignment. A straight incision of about $7 \mathrm{~cm}$ was made just lateral to the tibial crest to a point $5 \mathrm{~cm}$ distal to the tibial tuberosity. Anteromedialization was performed as described elsewhere [17]. The amount of anteriorization and medialization was decided by testing the position of the released tibial tuberosity during repeated flexion-extension movements. A second incision was then performed lateral to the patella; the patella was partially everted, the lesion debrided and the MACI technique performed as described elsewhere [9] (Fig. 2). The membrane was secured with fibrin glue (Tissucol ${ }^{\circledR}$ ) and a variable number of resorbable stitches, depending on defect site and the presence of a good defect margin. After ensuring haemostasis a suction drain was placed and the wound was closed.

\section{Postoperative rehabilitation}

All patients underwent the same rehabilitation program. The program is based on the fact that in knee flexion $>30^{\circ}$ the shear forces across the patella are always associated with a strong compression due to ligament tension, also in non-weightbearing joints [24]. Accordingly, $24 \mathrm{~h}$ after the operation patients were allowed active joint movement from $0^{\circ}$ to $20^{\circ}$ in a brace (to avoid increasing the patellofemoral joint reaction force), early full weightbearing with the knee in $0^{\circ}$ to $20^{\circ}$ of flexion, isometric quadriceps contractions and leg lifts. After 30 days the brace was
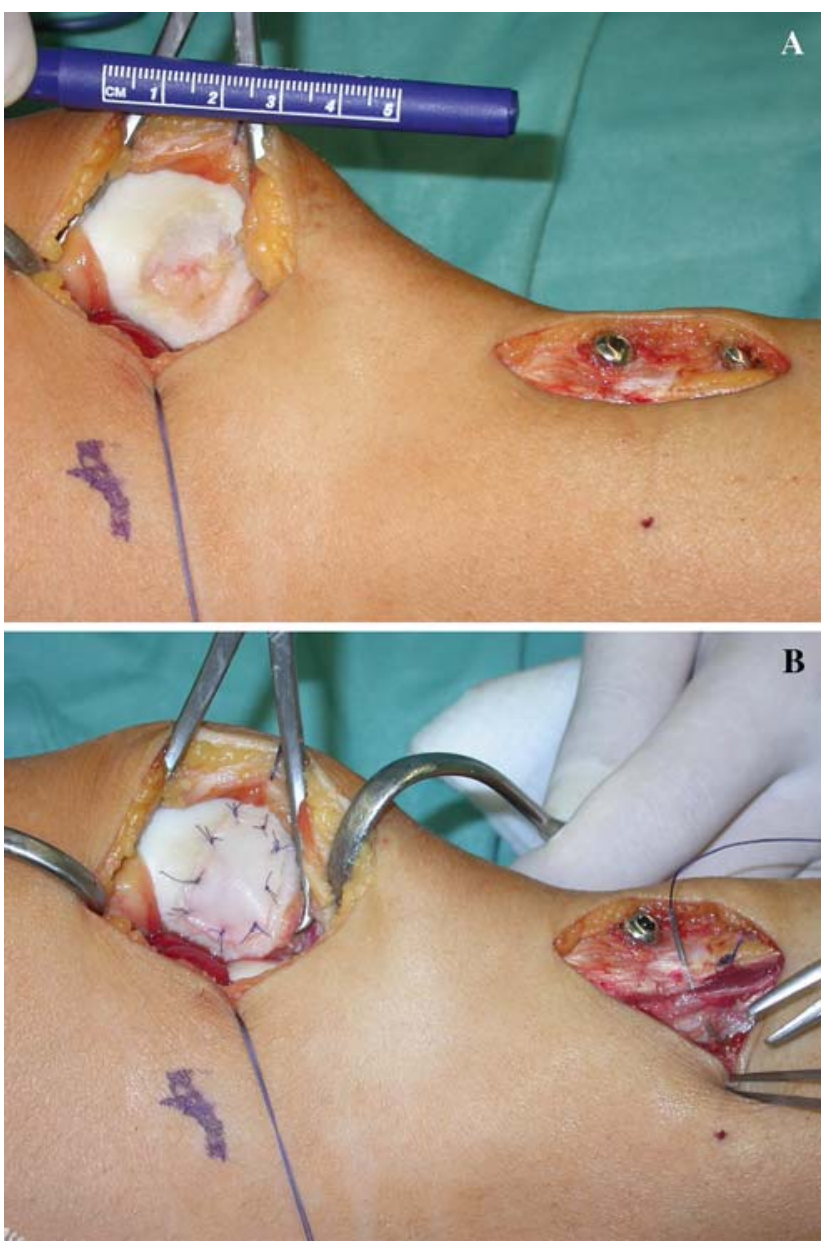

Fig. 2 Surgical access to the patella and distal tuberosity. Anteromedialization of the tibial tuberosity: a debridement of the patellar cartilage lesion; b suture of the membrane $\left(\mathrm{MACI}^{\circledR}\right)$ to the surrounding healthy cartilage and attachment with fibrin glue $\left(\right.$ Tissucol $^{\circledR}$ )

removed and knee flexion was progressively increased. At this time gentle cycling and swimming were began to restore the normal range of motion. High-impact sports were allowed at 8 months.

\section{Clinical evaluation}

Clinical evaluation was performed by the junior author (D. Enea). Patients were evaluated preoperatively and at 1,3 , 6, 12, 24 and 36 months using the Kujala Knee Score [30], the Tegner Activity Scale [47] and the Lysholm Knee Score [32]. The Modified Cincinnati Rating Scale [37, 41] was administered preoperatively and at 36 months and the Patient Satisfaction Survey (PSS) at 36 months [37]. The PSS includes four questions where patients are asked (1) about their state compared with before surgery (answers: much better, somewhat better, about the same, somewhat worse); (2) to rate their overall satisfaction (very satisfied, 
somewhat satisfied, neutral, very dissatisfied); (3) about the possibility to have the same surgery again (definitely yes, probably yes, uncertain, probably not) and (4) to rate outcome (excellent, good, fair, poor). Bilateral procedures were assessed separately for each knee.

\section{Statistical analysis}

A non-parametric approach was used given the small number of observations. Outcome measures were the percent variations $(\Delta \%)$ in pre- and postoperative Kujala, Tegner, Lysholm and Modified Cincinnati Rating Scale scores. Variations were analyzed according to lesion grade and type, and compared by means of the Wilcoxon ranksum test. Pre- and postoperative scores were compared with the Wilcoxon signed-ranks test.

Results were expressed as frequencies (absolute and percent) for categorical variables and as percentiles (median, 25th and 75th percentiles) for quantitative variables. A level of $P=0.05$ was considered as statistically significant.

\section{Results}

Twelve patients, six male and six female, with a median age of 31 years (25th to 75th percentiles: 25-35 years) were enrolled. Two patients had bilateral procedures. The knee was considered as the unit of observation. Overall, 14 patellar lesions were treated in eight right $(57 \%)$ and six left knees (43\%). There was one bifocal lesion. The median BMI was $23.2 \mathrm{~kg} / \mathrm{m}^{2}$ (25th to 75 th percentiles: $21.6-26$; range 19.4-26.4). The median lesion diameter was $4 \mathrm{~cm}^{2}$ (25th to 75 th percentiles: $3-5 \mathrm{~cm}^{2}$; range 3-9). There were $10(71.4 \%)$ grade IV and $4(28.6 \%)$ grade III lesions according to Outerbridge, and 7 (50\%) diffuse lesions, 5 (36\%) lesions of the medial facet and $2(14 \%)$ of the lateral facet according to Pidoriano et al. [45]. Patient data are shown in Table 1.

There were no major medical or surgical complications, either in the perioperative period or in the longer term. None of the patients were lost to follow-up. Two knees required screw removal during follow-up. No other additional surgical procedures were performed on the operated knees. Three patients had undergone previous surgical procedures in the operated knee, one partial medial meniscectomy and two lateral releases. None of the patients had clinically detectable calcaneus valgus or increased femoral anteversion; three female patients had valgus knee (femorotibial internal angle $>7^{\circ}$ ); four patients had Dejour type I trochlear dysplasia [13] and five had patella alta according to Insall and Salvati [27]. None of the three patients suffering from recurrent patellar dislocation experienced a feeling of instability or a dislocation episode during follow-up.

Pre- and postoperative Kujala, Tegner, Lysholm and Modified Cincinnati Rating Scale scores are reported in Fig. 3. All patients experienced significant improvement on all scales. The physician-rated Modified Cincinnati Rating Scale showed a median improvement of $200 \%$ (95\%CI: 100-400), from 2 points preoperatively (25th to 75 th percentiles: $2-4$ ) to 8 points postoperatively (25th to 75 th percentiles: 6-10). The Lysholm Knee Score displayed a median improvement of $57.3 \%$ (95\%CI 29.9-158), from 55 points (25th to 75 th percentiles: $47-74$ ) to 92.5 points postoperatively (25th to 75 th percentiles: $85-99$ ). The Tegner Activity Scale exhibited a median improvement of $300 \%$ (95\%CI 200-400), from 1 point (25th to 75th percentiles: $1-1$ ) to 4 points postoperatively (25th to 75 th percentiles: 4-5). The Kujala Score showed a median improvement of $63.7 \%$ (95\% CI 26.7-120.9) from 52 points (25th to 75th percentiles: $43-69$ ) to 88.5 points (25th to 75th percentiles: 85-95) (Table 1).

The main outcome measures were not significantly different when analyzed by lesion grade and type (Figs. 4, 5).

At the time of the preoperative evaluation 11 patients had a Tegner Activity Scale score of 1 (only able to perform sedentary work) and one patient had a score of 6 (basketball). At 36 months one patient had a score of 3 (swimming), 4 patients had a score of 4 (cycling, jogging), 4 patients had a score of 5 (competitive cycling, heavy labor), one patient had a score of 6 (basketball) and another patient had a score of 7 (competitive tennis). Overall, patient activity levels consistently improved, as demonstrated by the Tegner score increases. The median score rose from 1 (sedentary work) preoperatively to 4 (recreational sports: cycling and jogging) after the operation.

According to the PSS [37], patient satisfaction was high in 13/14 cases, with 50\% excellent and $43 \%$ good final outcomes and poor satisfaction in one case $(7 \%)$.

Two patients had second-look arthroscopy at 18 and 31 months, respectively, during screw removal. One patient, a man, complained of pain at the screw site at the time of the biopsy; the other, a woman, was dissatisfied with the aesthetic appearance of her tibial tuberosity, due to the slightly protruding screws. None of these patients had anterior knee pain related to the patellofemoral joint. After screw removal the man became asymptomatic in a few months.

\section{Discussion}

A small number of recent studies have addressed the effectiveness of the ACI or similar techniques in treating patellofemoral joint cartilage defects, documenting 
Table 1 Main characteristics of patients divided by surgical facility

\begin{tabular}{|c|c|c|c|c|c|c|c|}
\hline & \multicolumn{2}{|c|}{ Ancona $(n=8)$} & & \multicolumn{2}{|l|}{ Milan $(n=6)$} & \multicolumn{2}{|c|}{$P$-value (Fisher's exact test) } \\
\hline & $n$ & $\%$ & & $n$ & $\%$ & & \\
\hline Gender (male) & 5 & 62.5 & & 1 & 16.7 & \multicolumn{2}{|l|}{0.138} \\
\hline Side (right) & 4 & 50.0 & & 4 & 66.7 & \multicolumn{2}{|l|}{0.627} \\
\hline \multicolumn{8}{|c|}{ Grade (Outerbridge) } \\
\hline III & 2 & 25.0 & & 2 & 33.3 & \multicolumn{2}{|l|}{1} \\
\hline IV & 6 & 75.0 & & 4 & 66.7 & & \\
\hline \multicolumn{8}{|l|}{ Type [45] } \\
\hline I & 3 & 37.5 & & 4 & 66.7 & 0.627 & \\
\hline II & 3 & 37.5 & & 2 & 33.3 & & \\
\hline III & 2 & 25.0 & & 0 & 0.0 & & \\
\hline & Ancona & & Milan $(n$ & $=6)$ & $P$-value & Total & \\
\hline & Median & $\begin{array}{l}25 \text { th to } 75 \text { th } \\
\text { percentiles }\end{array}$ & Median & $\begin{array}{l}25 \text { th to } 75 \text { th } \\
\text { percentiles }\end{array}$ & & Median & $\begin{array}{l}25 \text { th to } 75 \text { th } \\
\text { percentiles }\end{array}$ \\
\hline Patient age & 32 & $25-37.5$ & 30 & $28-32$ & 0.57 & 31 & $28-35$ \\
\hline Lesion diameter & 4 & $3.5-4.5$ & 4 & $3-9$ & 0.841 & 4 & $3-5$ \\
\hline$K$ pre & 49 & $37-68$ & 58 & $48-79$ & 0.292 & 52 & $43-69$ \\
\hline$K$ post & 86.5 & $84-93$ & 92.5 & $87-100$ & 0.291 & 88.5 & $85-95$ \\
\hline$\Delta \% K$ & 76.9 & $36.8-121.4$ & 40.7 & $13.9-81.3$ & 0.198 & 63.7 & $26.7-107.5$ \\
\hline LY pre & 54.5 & $39-75.5$ & 55 & $47-72$ & 1 & 55 & $47-74$ \\
\hline LY post & 88.5 & $82.5-99$ & 94 & $85-100$ & 0.702 & 92.5 & $85-99$ \\
\hline$\Delta \% \mathrm{LY}$ & 57.3 & $31.8-119.5$ & 56.2 & $27.3-100$ & 0.949 & 57.3 & $29.9-100$ \\
\hline$T$ pre & 1 & $1-1$ & 1 & $1-1$ & 0.643 & 1 & $1-1$ \\
\hline$T$ post & 4 & $3.5-4$ & 5 & $5-6$ & 0.065 & 4 & $4-5$ \\
\hline$\Delta \% T$ & 300 & $250-350$ & 300 & $200-400$ & 0.885 & 300 & $200-400$ \\
\hline$C$ pre & 2 & $2-4$ & 2 & $2-3$ & 0.713 & 2 & $2-4$ \\
\hline$C$ post & 7 & $6-8$ & 9 & $8-10$ & 0.245 & 8 & $6-10$ \\
\hline$\Delta \% \mathrm{C}$ & 200 & $75-300$ & 100 & $250-400$ & 0.699 & 200 & $100-400$ \\
\hline
\end{tabular}

Grade lesion grade according to Outerbridge, Type lesion type according to Pidoriano et al. [42], $K$ Kujala Score, $L Y$ Lysholm Knee Score, $T$ Tegner Activity Scale; $C$ Modified Cincinnati Rating Scale, Pre preoperative, Post postoperative, $\Delta \%$ percent variation, Ancona patients operated on in Ancona, Milan patients operated on in Milan

variable and heterogeneous outcomes. Brittberg et al.'s early results achieved only $29 \%$ good to excellent outcomes [7]. Lorentzon et al. reported $96 \%$ good to excellent results in isolated patellar cartilage defects treated by periosteal transplantation, using a particular periosteal suture, associated with continuous passive motion in the immediate postoperative period. They did not use chondrocyte cultures and did not perform realignment [31]. Peterson and co-workers [43, 44] obtained $76 \%$ good to excellent results at 10 years by associating, where necessary, an operative realignment of the extensor mechanism. Minas and Bryant [37] described $71 \%$ good to excellent results in a fairly heterogeneous population. Gobbi et al. [20], using a hyaluronic acid synthetic scaffold instead of the periosteal flap, obtained $90 \% \mathrm{~A}$ and B results according to the IKDC knee ligament standard evaluation at 2 years.
In a retrospective cohort study Niemeyer et al. compared the outcomes of periosteal patch-covered conventional ACI, collagen membrane-covered ACI and matrix-associated $\mathrm{ACI}$ in treating 70 patients with retropatellar cartilage defects. Patients with patellofemoral malalignment (assessed on axial knee x-rays) were excluded in order to obtain a homogeneous sample. The authors reported good to excellent result in approximately $70 \%$ of patients and found larger lesions, lesions on the medial patellar facet and diffuse lesions to have a poorer prognosis than smaller lesions and lesions on the lateral patellar facet. The clinical outcomes of the three surgical techniques used were no significantly different [39].

In a recent study, Henderson et al. [23] compared two groups of 22 patients one with patellofemoral malalignment and patellar cartilage defects and the other with patellar 
Fig. 3 Pre- and postoperative Kujala Score $(K)$, Lysholm Knee Score $(L Y)$, Tegner Activity Scale $(T)$ and Modified Cincinnati Rating Scale $(C)$ scores
Fig. 4 Percent variations $(\Delta \%)$ in pre- and postoperative Kujala Score $(K)$, Lysholm Knee Score $(L Y)$, Tegner Activity Scale $(T)$ and Modified Cincinnati Rating Scale $(C)$ scores in relation to lesion grade
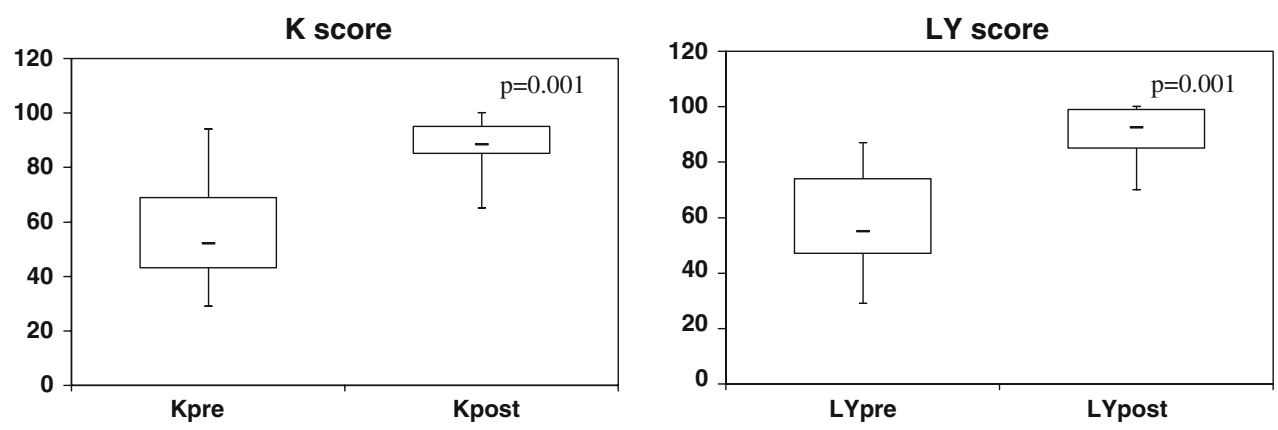

T score

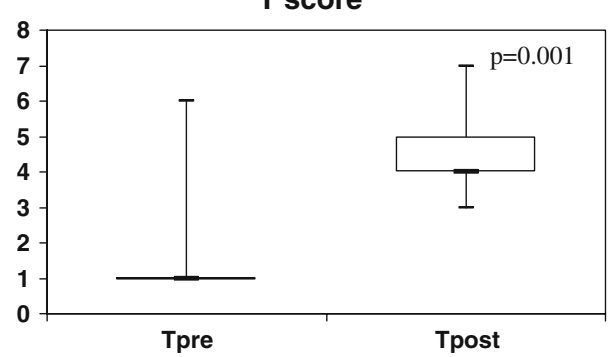

C score
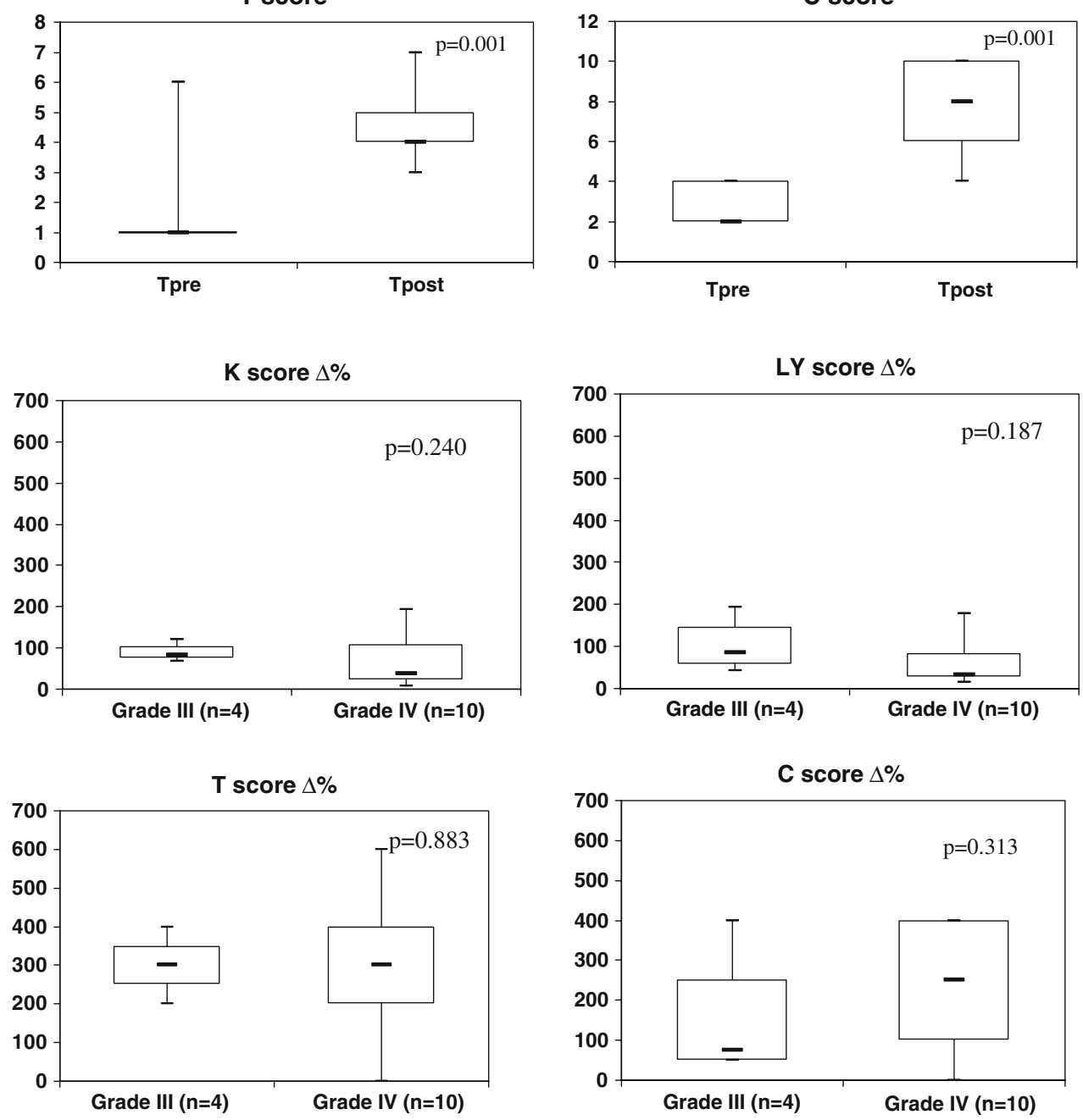

cartilage defects only. The two groups were treated with ACI combined with distal or proximal realignment or with ACI alone, respectively. The authors reported $86 \%$ good to excellent outcomes in the first group versus $55 \%$ in the second. They ascribed the difference to unloading due to the distal osteotomy or to incorrect preoperative evaluation of the extent of malalignment in the patients of the second group, and concluded that patellofemoral joint unloading with a realignment procedure is desirable to maximize the clinical outcome of ACI, even when no tracking anomalies are identified clinically. Their reoperation rate was $52 \%$ (10/22 and 13/22 patients, respectively).

In a study similar to the present one, Farr [15] considered 38 patients with patellar and/or trochlear cartilage lesions, of whom 28 underwent distal realignment prior to or simultaneously with ACI. Thirteen of the 28 patients had isolated patellar cartilage lesions, but no data were provided as to the type of malalignment treated, and 
Fig. 5 Evaluation of percent variations $(\Delta \%)$ in pre- and postoperative Kujala Score $(K)$, Lysholm Knee Score $(L Y)$, Tegner Activity Scale $(T)$ and Modified Cincinnati Rating Scale $(C)$ scores in relation to lesion type
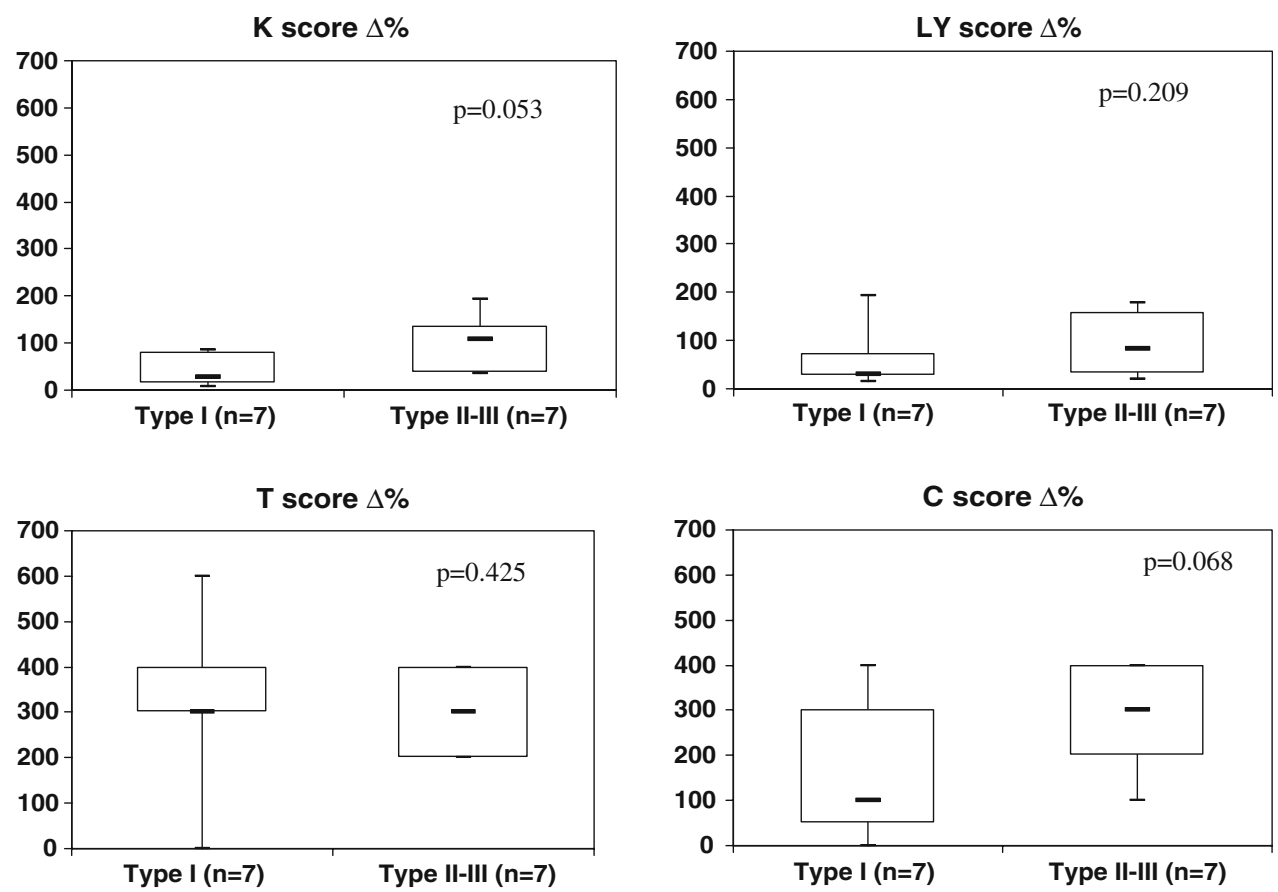

patellofemoral congruence was assessed only by a lateral radiograph and a Merchant axial view, without specification of the amount of subluxation. The two patients with a tilted and subluxated patella and those with no clear subluxation received patellar realignment followed by a rehabilitation program permitting earlier range of motion recovery compared with our patients; our rehabilitation protocol was more aggressive in allowing earlier full weightbearing. Overall, 25 of Farr's 38 patients required additional surgery, 18 for procedures other than screw removal. Nonetheless, their median Modified Cincinnati scores ranged from 3 to 6 and the Lysholm score from 56 to 86 .

The relative effectiveness of realignment and ACI in treating malalignment associated with patellar cartilage lesions is at present difficult to assess, due to the fact that the outcomes of ACI alone and of ACI with an extensor mechanism correction have often been reported together. In addition, the lack of a control group does not allow to establish whether one or the other procedure alone can provide both pain relief and functional improvement, but ethical reasons suggest that both conditions should be treated.

Our study focused on a small subgroup of patients with anterior knee pain and tilted and subluxated patella with TT-TS $>20 \mathrm{~mm}$ on preoperative CT scans associated with large, isolated, patellar cartilage lesions. In such cases the surgical approach should address both conditions, because transfer of the tibial tuberosity alone cannot solve the cartilage problem and could thus fail to provide pain relief [45]. On the other hand, it is disputed whether cartilage defects should be repaired without addressing the biomechanical imbalance [23].

A combination of MACI and transfer of the tibial tuberosity achieved good results in this selected group of patients, with significant increases in all outcome scales. These data are in line with those from similar studies, none of which however provide detailed information on the type of patients included or the type of malalignment treated. We failed to find significant percent variations in outcome scores between patients with Outerbridge grade III and grade IV lesions. This however is not surprising, because both lesion types are end-stage cartilage lesions associated with an altered load distribution across the subchondral bone [48].

Similarly, patients with diffuse lesions showed not significantly different percent variations in outcome scores compared with patients whose lesions were located on the lateral or the medial patellar facet. In this case, the data reported by Pidoriano et al. [45] and Niemeyer et al. [39] had led us to expect some differences, but our smaller sample size may have affected the result, making such difference undetectable. We did not analyze the main outcome measures by lesion size. In literature some studies have reported no association between defect size and the clinical outcome [29], while other studies had reported a significant association [39].

According to the PSS, results were excellent in $50 \%$ of cases and good in 43\% (overall 13/14 patients; 93\%). One patient $(7 \%)$ was not satisfied with the outcome of the 
procedure. This patient was the one with the highest activity level before the operation (6 points according to the Tegner Score) and the highest expectations. His dissatisfaction was due to incomplete pain relief despite maintaining the same level of functional activity.

The study suffers from some limitations. First of all, the small sample and the 36-month follow-up do not prove that the technique is effective in the longer term. Secondly, non-blinded postoperative assessment may have led to overestimation of favorable outcomes. Finally, the study lacks a control group, although not treating one or the other condition would of course have posed an ethical problem.

Overall, the present data support the case for combining distal realignment and patellar ACI to treat large, isolated patellar cartilage lesions due to severe malalignment. Larger series with longer follow-up are needed better to establish the effectiveness and long-term durability of this therapeutic strategy.

Acknowledgments We are grateful to Prof. F. Carle and Dr R. Gesuita (Istituto di Medicina Clinica e Biotecnologie Applicate, Polytechnic University of Marche) for the statistical studies. The authors also wish to thank Dr S. Modena for language revision and Ms S. Manzotti for preparing the histological sections.

Open Access This article is distributed under the terms of the Creative Commons Attribution Noncommercial License which permits any noncommercial use, distribution, and reproduction in any medium, provided the original author(s) and source are credited.

\section{References}

1. Aglietti P, Giron F, Cuomo P (2006) Insall \& Scott Surgery of the Knee, 4th edn. Churchill Livingstone, Philadelphia, p 848

2. Arendt EA, Fithian DC, Cohen E (2002) Current concepts of lateral patella dislocation. Clin Sports Med 21(3):499-519

3. Bartlett W, Skinner JA, Gooding CR, Carrington RW, Flanagan AM, Briggs TW, Bentley G (2005) Autologous chondrocyte implantation versus matrix-induced autologous chondrocyte implantation for osteochondral defects of the knee: a prospective, randomised study. J Bone Joint Surg Br 87(5):640-645

4. Beaconsfield T, Pintore E, Maffulli N, Petri G (1994) Radiological measurements in patellofemoral disorders: a review. Clin Orthop Relat Res 308:18-28

5. Bellemans J, Cauwenberghs F, Witvrouw E, Brys P, Victor J (1997) Anteromedial tibial tubercle transfer in patients with chronic anterior knee pain and a subluxation-type patellar malalignment. Am J Sports Med 25(3):375-381

6. Bentley G, Biant LC, Carrington RW, Akmal M, Goldberg A, Williams AM, Skinner JA, Pringle J (2003) A prospective, randomised comparison of autologous chondrocyte implantation versus mosaicplasty for osteochondral defects in the knee. J Bone Joint Surg Br 85(2):223-230

7. Brittberg M, Lindahl A, Nilsson A, Ohlsson C, Isaksson O, Peterson L (1994) Treatment of full-thickness cartilage defects in the human knee with cultured autologous chondrocytes. N Engl J Med 331:889-895
8. Chen FS, Frenkel SR, Di Cesare PE (1999) Repair of articular cartilage defects: part I. Basic science of cartilage healing. Am J Orthop 28(1):31-33

9. Cherubino P, Ronga M, Grassi FA, Bulgheroni P, Cherubino M (2006) Surgical transplantation technique. In: Zanasi S, Brittberg M, Maracci M (eds) Basic science, clinical repair and reconstruction of articular cartilage defects: current status and prospects. Data Trace Publishing Co, Brooklandville, p 533

10. Curl WW, Krome J, Gordon ES, Rushing J, Smith BP, Poehling GG (1997) Cartilage injuries: a review of 31, 516 knee arthroscopies. Arthroscopy 13(4):456-460

11. Davies AP, Vince AS, Shepstone L, Donell ST, Glasgow MM (2002) The radiologic prevalence of patellofemoral osteoarthritis. Clin Orthop Relat Res (402):206-212

12. Dejour H, Walch G, Nove-Josserand L, Guier C (1994) Factors of patellar instability: an anatomic radiographic study. Knee Surg Sports Traumatol Arthrosc 2(1):19-26

13. Dejour H, Walch G, Neyret P, Adeline P (1990) La dysplasie de la trochlée femorale. Rev Chir Orthop Reparatrice Appar Mot 96:45

14. Desio SM, Burks RT, Bachus KN (1998) Soft tissue restraints to lateral patellar translation in the human knee. Am J Sports Med 26(1):59-65

15. Farr J (2007) Autologous chondrocyte implantation improves patellofemoral cartilage treatment outcomes. Clin Orthop Relat Res 463:187-194

16. Fithian DC, Paxton EW, Cohen AB (2004) Indications in the treatment of patellar instability. J Knee Surg 17(1):47-56

17. Fulkerson JP, Becker GJ, Meaney JA, Miranda M, Folcik MA (1990) Anteromedial tibial tubercle transfer without bone graft. Am J Sports Med 18(5):490-496

18. Fulkerson JP, Shea KP (1990) Disorders of patellofemoral alignment. J Bone Joint Surg Am 72(9):1424-1429

19. Gobbi A, Nunag P, Malinowski K (2005) Treatment of full thickness chondral lesions of the knee with microfracture in a group of athletes. Knee Surg Sports Traumatol Arthrosc 13(3):213-221

20. Gobbi A, Kon E, Berruto M, Francisco R, Filardo G, Marcacci M (2006) Patellofemoral full-thickness chondral defects treated with Hyalograft-C: a clinical, arthroscopic, and histologic review. Am J Sports Med 34(11):1763-1773

21. Guzzanti V, Gigante A, Di Lazzaro A, Fabbriciani C (1994) Patellofemoral malalignment in adolescents. Computerized tomographic assessment with or without quadriceps contraction. Am J Sports Med 22(1):55-60

22. Hangody L, Kish G, Kárpáti Z, Udvarhelyi I, Szigeti I, Bély M (1998) Mosaicplasty for the treatment of articular cartilage defects: application in clinical practice. Orthopedics 21(7):751-756

23. Henderson IJ, Lavigne P (2006) Periosteal autologous chondrocyte implantation for patellar chondral defect in patients with normal and abnormal patellar tracking. Knee 13(4):274-279

24. Hungerford DS, Barry M (1979) Biomechanics of the patellofemoral joint. Clin Orthop Relat Res (144):9-15

25. Hunter DJ, Zhang YQ, Niu JB, Felson DT, Kwoh K, Newman A, Kritchevsky S, Harris T, Carbone L, Nevitt M (2007) Patella malalignment, pain and patellofemoral progression: the Health ABC Study. Osteoarthritis Cartilage 15(10):1120-1127

26. Insall J, Falvo KA, Wise DW (1976) Chondromalacia patellae. A prospective study. J Bone Joint Surg Am 58(1):1-8

27. Insall J, Salvati E (1971) Patella position in the normal knee joint. Radiology 101(1):101

28. Knutsen G, Engebretsen L, Ludvigsen TC, Drogset JO, Grøntvedt T, Solheim E, Strand T, Roberts S, Isaksen V, Johansen O (2004) Autologous chondrocyte implantation compared with microfracture in the knee. A randomized trial. J Bone Joint Surg Am 86-A(3):455-464 
29. Knutsen G, Drogset JO, Engebretsen L, Grøntvedt T, Isaksen V, Ludvigsen TC, Roberts S, Solheim E, Strand T, Johansen O (2007) A randomized trial comparing autologous chondrocyte implantation with microfracture. Findings at five years. J Bone Joint Surg Am 89:2105-2112

30. Kujala UM, Jaakkola LH, Koskinen SK, Taimela S, Hurme M, Nelimarkka O (1993) Scoring of patellofemoral Disorders. Arthroscopy 9(2):159-163

31. Lorentzon R, Alfredson H, Hildingsson C (1998) Treatment of deep cartilage defects of the patella with periosteal transplantation. Knee Surg Sports Traumatol Arthrosc 6(4):202-208

32. Lysholm J, Gillquist J (1982) Evaluation of knee ligament surgery results with special emphasis on use of a scoring scale. Am J Sports Med 10:150-154

33. Maquet P (1976) Advancement of the tibial tuberosity. Clin Orthop Relat Res (115):225-230

34. Mc Ginley BJ, Cushner FD, Scott WN (1999) Debridement arthroscopy. 10-year followup. Clin Orthop Relat Res (367): 190-194

35. Messner K, Maletius W (1996) The long-term prognosis for severe damage to weight-bearing cartilage in the knee: a 14-year clinical and radiographic follow-up in 28 young athletes. Acta Orthop Scand 67(2):165-168

36. Miller BJ, LaRochelle PJ (1986) The treatment of patellofemoral pain by combined rotation and elevation of the tibial tubercle. $J$ Bone Joint Surg Am 68(3):419-423

37. Minas T, Bryant T (2005) The role of autologous chondrocyte implantation in the patellofemoral joint. Clin Orthop Relat Res (436):30-39

38. Morshuis WJ, Pavlov PW, de Rooy KP (1990) Anteromedialization of the tibial tuberosity in the treatment of patellofemoral pain and malalignment. Clin Orthop Relat Res (255):242-250

39. Niemeyer P, Steinwachs M, Erggelet C, Kreuz PC, Kraft N, Köstler W, Mehlhorn A, Südkamp NP (2007) Autologous chondrocyte implantation for the treatment of retropatellar cartilage defects: clinical results referred to defect localisation. Arch Orthop Trauma Surg (online 21 Aug 2007)
40. Noll BJ, Ben-Itzhak I, Rossouw P (1988) Modified technique for tibial tubercle elevation with realignment for patellofemoral pain. A preliminary report. Clin Orthop Relat Res (234):178-182

41. Noyes FR, Barber SD, Moolar LA (1989) A rationale for assessing sports activity levels and limitations in knee disorders. Clin Orthop Relat Res 246:238-249

42. Peterson L, Karlsson J, Brittberg M (1988) Patellar instability with recurrent dislocation due to patellofemoral dysplasia. Results after surgical treatment. Bull Hosp Jt Dis Orthop Inst 48(2):130-139

43. Peterson L, Minas T, Brittberg M, Nilsson A, Sjögren-Jansson E, Lindahl A (2000) Two- to 9-year outcome after autologous chondrocyte transplantation of the knee. Clin Orthop Relat Res (374):212-234

44. Peterson L, Brittberg M, Kiviranta I, Akerlund EL, Lindahl A (2002) Autologous chondrocyte transplantation. Biomechanics and long-term durability. Am J Sports Med 30(1):2-12

45. Pidoriano AJ, Weinstein RN, Buuck DA, Fulkerson JP (1997) Correlation of patellar articular lesions with results from anteromedial tibial tubercle transfer. Am J Sports Med 25(4):533-537

46. Schutzer SF, Ramsby GR, Fulkerson JP (1986) The evaluation of patellofemoral pain using computerized tomography. A preliminary study. Clin Orthop Relat Res 204:286-293

47. Tegner Y, Lysholm J (1985) Rating system in the evaluation of knee ligament injuries. Clin Orthop Relat Res 198:43-49

48. Thambyah A, Broom N (2007) On how degeneration influences load-bearing in the cartilage-bone system: a microstructural and micromechanical study. Osteoarthritis Cartilage 15:1410-1423

49. Trillat A, Dejour H, Couette A (1964) Diagnosis and treatment of recurrent dislocation of the patella. Rev Chir Orthop Reparatrice Appar Mot 50:813-824

50. Widuchowski W, Widuchowski J, Trzaska T (2007) Articular cartilage defects: study of 25, 124 knee arthroscopies. Knee 14(3):177-182 